\title{
Multiple cardiac complications after adjuvant therapy for breast cancer: the importance of echocardiography. A case report and review of the literature.
}

\author{
Adriana Luminita Gurghean, Ilinca Savulescu-Fiedler, Anca Mihailescu
}

Department of Cardiology, Coltea Clinical Hospital, "Carol Davila" Universtity of Medicine and Pharmacy, Bucharest, Romania

\begin{abstract}
Cardiovascular complications induced by adjuvant cancer therapies may become symptomatic after many years, being responsible for increased morbidity and mortality in long-term survivors. We report a case of a 54-year old female admitted for severe heart failure induced by myocardial and valvular damage after postoperative adjuvant therapy for left breast cancer 6 years ago. Her recent history revealed nonST elevation myocardial infarction in the absence of significant cardiovascular risk factors. Transthoracic echocardiography, tissue Doppler imaging and speckle-tracking imaging revealed severe biventricular systolic dysfunction, severe mitral and tricuspid regurgitation and severe pulmonary hypertension.

Keywords: cancer adjuvant therapies, cardiovascular complications, echocardiography, tissue Doppler imaging, speckle tracking.
\end{abstract}

\section{Introduction}

Breast cancer is the most common type of cancer in women, with continuous increasing incidence. Adjuvant postoperative therapies for breast cancer are, with no doubt, responsible for improved outcome and increased survival by reducing the risk of local recurrences by about two-thirds [1]. Nevertheless, most of these therapies and their combination can potentially cause various acute and late cardiovascular complications that are considered the leading cause for increased morbidity and mortality in cancer survivors, not related to the neoplasia itself [2]. Cardiac damage may not be apparent until years after the end of adjuvant cancer therapies, but when symptomatic, the evolution is rapid and the prognosis is significantly influenced.

Received 20.07.2016 Accepted 18.08.2016

Med Ultrason

2017, Vol. 19, No 1, 117-120

Corresponding author: Adriana Luminita Gurghean

Department of Cardiology, Coltea Clinical

Hospital, "Carol Davila" Universtity of

Medicine and Pharmacy, Bucharest, Romania

1, I.C. Bratianu street, Sector 3

030171, Bucharest, Romania

Phone +40723336692

E-mail: adrianagurghean@yahoo.com
We present a case of severe heart failure secondary to complex cardiac damage 6 years postradio- and chemotherapy for breast cancer in a patient with no history of cardiovascular risk factors or cardiovascular disease at the time of the oncological treatment.

\section{Case report}

A 54-year-old female presented with inspiratory dyspneea at moderate effort in the last month and paroxistic nocturnal dyspneea in the last week (first episode). She had a history of nonST myocardial infarction and stenting of the circumflex artery 18 months before. Six years before she had surgery for left breast cancer and adjuvant oncological therapy consisting of chemotherapy (no documents regarding the treatment regimen) and radiotherapy (a total dose of 50 Gy). The patient had no cardiovascular disease prior to the breast cancer diagnosis and no significant cardiovascular risk factors.

Physical examination revealed left anterior thoracic scar, postmastectomy, fine crackles in both lung bases and no signs of systemic congestion. Cardiac examination revealed regular cardiac rhythm, left S3 sound and a holosystolic murmur of mitral regurgitation. Chest ra- 
diography revealed signs of pulmonary stasis and normal cardiac dimensions. Standard electrocardiography (ECG) revealed sinus rhythm, with no acute morphological changes. The laboratory tests were normal, with the exception of an increased value of the N-terminal-pro brain natriuretic peptide (NT-proBNP) of $524 \mathrm{pg} / \mathrm{ml}$ (reference values: $10-157 \mathrm{pg} / \mathrm{ml}$ ). Oncological evaluation found no signs of activity of the disease. Specific tumoral serum markers were between normal values.

Conventional transthoracic echocardiography revealed mild left ventricular and atrial dilation, normal right atrial and ventricular cavities, but with right ventricular hypertrophy. Valvular morphology revealed important fibrotic lesions of the posterior mitral leaflet, with severe restriction of its mobility and severe mitral regurgitation (fig 1a). Severe tricuspid regurgitation and a calculated systolic pulmonary artery pressure of 69 $\mathrm{mmHg}$ were found, but no pericardial abnormalities. The patient had diffuse hipokinesia and severe global systolic dysfunction of the left ventricle, with a calculated left ventricular ejection fraction of 38\% (Simpson method). Severe left ventricular systolic dysfunction was confirmed by tissue Doppler imaging which revealed decreased peak systolic annular velocities in all ventricular walls $\left(\mathrm{S}^{\prime}=5 \mathrm{~cm} / \mathrm{s}\right)$ (fig $1 \mathrm{~b}$ ) and by speckle-tracking deformation imaging (strain and strain rate). Important changes of the global longitudinal strain (GLS \%) of the left ventricle, the lowest values found were $-13.2 \%$ for the longitudinal axis view (normal values for age and gender are $-20 \%$ ) (fig $2 a$ ), with an average value of the global longitudinal peak strain of $-15.2 \%$ (fig $2 b$ ) were detected. Moderate right ventricular systolic dysfunction was also found, with reduced tricuspid annular plane systolic excursion, TAPSE $=14 \mathrm{~mm}$ (normal values $24 \pm 3.5$ $\mathrm{mm}$ ), and reduced peak systolic tricuspid annular velocity at tissue Doppler imaging $-\mathrm{Sa}=14 \mathrm{~cm} / \mathrm{s}$ (normal values $18.1 \pm 2.3 \mathrm{~cm} / \mathrm{s}$ ) and right ventricle diastolic dysfunction $\left(E^{\prime} / A^{\prime}\right.$ ratio $\left.=0.48\right)$, in the absence of right ventricular morphological changes.

During hospitalization, the patient received specific treatment for heart failure with favorable outcome and had no episodes of angina. At discharge, the patient was referred to cardiac surgery for the evaluation of the opportunity of atrioventricular valvular reconstruction.

\section{Discussions}

The particularities of this case are: the occurrence of an acute coronary event in a relatively young female, in the absence of cardiovascular risk factors and the relatively short time after chemo- and radio-therapy in developing the first episode of heart failure. The severity

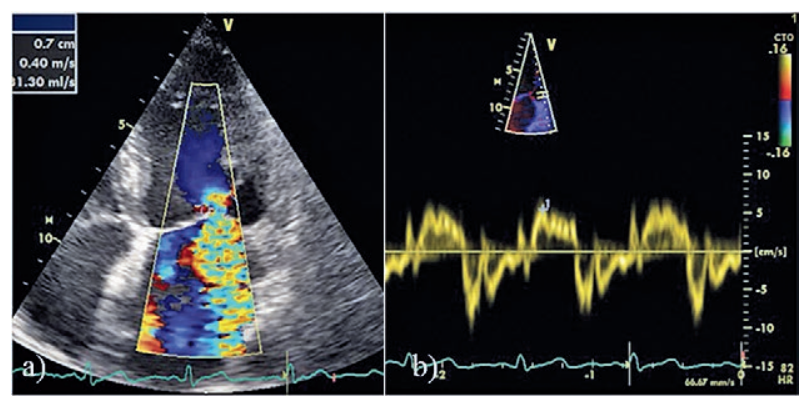

Fig 1. a) Apical 4 chamber view showing severe mitral regurgitation with an increased proximal isovelocity surface area (PISA) of $7 \mathrm{~mm}$; b) Tisue Doppler imaging at the lateral left ventricular wall showing severely reduced myocardial peak systolic annular velocity S' (cm/sec).

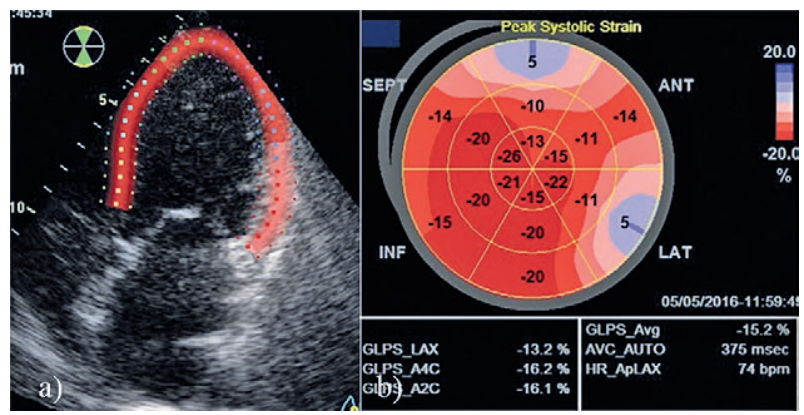

Fig 2. a) Global Longitudinal Strain imaging of the left ventricle showing reduced average value of peak systolic strain; b) Average global longitudinal strain of the left ventricle (reduced average values).

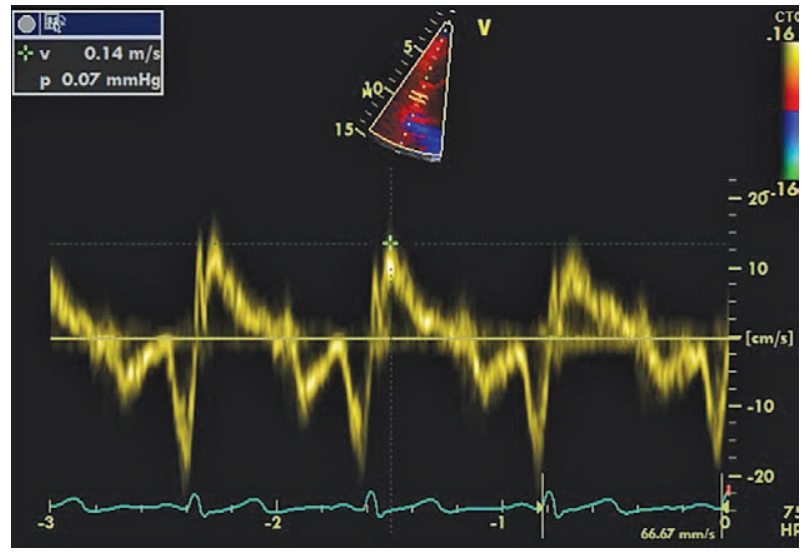

Fig 3. Tisue Doppler imaging at the lateral wall of the right ventricle showing reduced myocardial peak systolic velocity values $\mathrm{Sa}(\mathrm{cm} / \mathrm{sec})$ and diastolic dysfunction (reversed E'/A' ratio).

of cardiac morphological and functional changes found on ultrasound examination should also be noted. In the absence of an ultrasound cardiac examination, the importance and the character of the heart lesions, as well as the main etiology of heart failure (in a patient with 
a coronary event and chest irradiation) could not have been appreciated and, as a consequence, the therapeutic plan could not have been established. In our opinion, left ventricular dysfunction might be partially explained by the coronary event mentioned in the recent medical history of the patient, another potential mechanism being the severe myocardial fibrosis induced by radiotherapy. Left thoracic radiation might also explain the other abnormalities found on echocardiography - systolic right ventricular dysfunction in the absence of ventricular dilatation and valvular fibrosis with severe mitral and tricuspid regurgitations.

In this patient, in the presence of a severe mitral regurgitation, there was a modest dilatation of the left heart cavities which is another argument for fibrosis of the cardiac walls as a cause. In this context there was an important global hipokinesia leading to heart failure and severe pulmonary hypertension.

The benefic effect of radio- and chemo- therapy on survival is counterbalanced by the risk of side effects, among which cardiac damage is the most severe $[3,4]$. Radiation induced heart disease (RIHD) is a heterogeneous condition and includes pericarditis, myocardial fibrosis and cardiomyopathy, coronary artery disease, valvular disease and arrhythmias [5,6]. Patients with breast cancer (especially left breast cancer) and Hodgkin disease represent the largest population exposed to chest radiation [7].

Pathological changes in coronary arteries, induced by radiation are characterized by microvascular damage, inflammation and fibrosis. In medium and large arteries, morphological changes are quite similar to atherosclerosis [8], but in small arteries there is often subendotelial fibrosis, accumulation of acellular collagen in the media and of foam cells in the intima [9]. Dose of radiotherapy over 8 Gy are associated with increased size and number of lesions in major arteries and the resulting plaques are more unstable and prone to rupture $[10,11]$. Arterial fibrosis is considered an independent risk factor for longterm cardiovascular disease in early stage breast cancer. High-dose exposure is related to cardiac morbidity, including coronary artery disease but there is no consensus regarding a protective low-dose threshold.

Myocardial damage occurs after capillary loss from microvascular changes induced by radiotherapy because of subsequent hipoxia. Histological studies also revealed inflammatory and prothrombotic changes and progressive fibrosis replacing the myocardial tissue. There is an increase in both type I and type III collagen in irradiated hearts that leads to a decrease in tissue elasticity and distensibility and finally to a reduction in the ejection fraction and cardiac output [12]. The clinical expression of these changes is heart failure.
Postradiotherapy valvular disease is less well characterized compared to the vascular, myocardial and pericardial diseases, although valvular damage is frequently seen in these patients. Valvular fibrosis is not related to microvascular damage as heart valves are avascular. Post mortem analysis showed evidence of valvular fibrosis and dysfunction in up to $81 \%$ of patients and with no chronic inflammation $[13,14]$. The most frequent valvular dysfunction is valvular incompetence. A mean dose of $46 \mathrm{~Gy}$ is related to the presence of valvular fibrosis. It is appreciated that in most patients there is a significant latent period before the development of symptoms. Our patient was irradiated with a total dose of 50 Gy which may explain the magnitude and precocity of her heart lesions.

Identifying patients with high risk for cardiac damage due to cancer therapy is very important and cardiac imaging evaluation of patients before, during and after therapy is mandatory, along with clinical evaluation. Young age and left anterior thoracic radiation are considered significant risk factors for cardiac damage and these patients should be careful followed in order to detect subclinical changes. The most useful imaging technique is echocardiography because of its large availability, easy repeatability, accuracy and safety. It allows a comprehensive evaluation of cardiac valves, dimensions, systolic and diastolic function and of the aorta and pericardium [15]. New techniques like global longitudinal speckle tracking are especially useful for detecting subtle myocardial dysfunction in patients with apparently normal ventricular systolic function (normal ejection fraction) [16]. Therefore, these methods are now included in the follow-up protocols for patients that receive chemotherapy and/or radiotherapy [17].

\section{References}

1. Siegel R, Ma J, Zou Z, Jemal A. Cancer statistics, 2014. CA Cancer J Clin 2014;64:9-29.

2. Bouillon K, Haddy N, Delaloge S, et al. Long-term cardiovascular mortality after radiotherapy for breast cancer. $\mathrm{J}$ Am Coll Cardiol 2011;57:445-452.

3. Onitilo AA, Engel JM, Stankowski RV. Cardiovascular toxicity associated with adjuvant trastuzumab therapy: prevalence, patient characteristics and risk factors. Ther Adv Drug Saf 2014; 5:154-166.

4. Lancellotti P, Nkomo VT, Badano LP, et al. Expert consensus for multi-modality imaging evaluation of cardiovascular complications of radiotherapy in adults: a report from the European Association of Cardiovascular Imaging and the American Society of Echocardiography. Eur Heart J Cardiovasc Imaging 2013;14:721-740.

5. Darby SC, Ewertz M, McGale P, et al. Risk of ischemic heart disease in women after radiotherapy for breast cancer. N Engl J Med 2013;368:987-998. 
6. Zellars R, Bravo PE, Tryggestad E, et al. SPECT analysis of cardiac perfusion changes after whole-breast/chest wall radiation therapy with or without active breathing coordinator: results of a randomized phase 3 trial. Int J Radiat Oncol Biol Phys 2014;88:778-785.

7. Meyer R, Gospodarowicz M, Connors J, et al; NCIC Clinical Trials Group; Eastern Cooperative Oncology Group. ABVD alone versus radiation-based therapy in limited-stage Hodgkin's lymphoma. N Engl J Med 2012;366:399-408.

8. Madan R, Benson R, Sharma DN, Julka PK, Rath GK. Radiation induced heart disease: Pathogenesis, management and review literature. J Egypt Natl Canc Inst 2015;27:187193.

9. Andratschke N, Maurer J, Molls M, Trott KR. Late radiation-induced heart disease after radiotherapy. Clinical importance, radiobiological mechanisms and strategies of prevention. Radiother Oncol 2011;100:160-166.

10. Weintraub NL, Jones WK, Manka D. Understanding radiation-induced vascular disease. J Am Coll Cardiol 2010;55:1237-1239.

11. Taunk NK, Haffty BG, Kostis JB, Goyal S. Radiation-induced heart disease: Pathologic abnormalities and putative mechanisms. Front Oncol 2015;5:39.
12. Darby SC, Ewertz M, McGale P, et al. Risk of ischemic heart disease in women after radiotherapy for breast cancer. N Engl J Med 2013;368:987-998.

13. Gujral DM, Lloyd G, Bhattacharyya S. Radiation-induced valvular heart disease. Heart 2016;102:269-276.

14. Desai MY, Wu W, Masri A, et al. Increased aorto-mitral curtain thickness independently predicts mortality in patients with radiation-associated cardiac disease undergoing cardiac surgery. Ann Thorac Surg 2014;97:1348-1355.

15. Plana JC, Galderisi M, Barac A, et al. Expert consensus for multimodality imaging evaluation of adult patients during and after cancer therapy: a report from the American Society of Echocardiography and the European Association of Cardiovascular Imaging. J Am Soc Echocardiogr 2014;27:911-939.

16. Thavendiranathan P, Poulin F, Lim KD, Plana JC, Woo A, Marwick TH. Use of myocardial strain imaging by echocardiography for the early detection of cardiotoxicity in patients during and after cancer chemotherapy: a systematic review. J Am Coll Cardiol 2014;63:2751-2768.

17. Mor-Avi V, Lang RM. Is echocardiography reliable for monitoring the adverse cardiac effects of chemotherapy? J Am Coll Cardiol 2013;61:85-87. 